\title{
The Game: mutaciones didácticas desde el ultramundo
}

\author{
Reseña del libro The Game de Alessandro Baricco (2019). \\ Barcelona: Anagrama. Colección Argumentos, 333 pp. Traducción: Xavier González Rovira
}

\section{Miriam Kap}

\author{
Universidad Nacional de Mar del Plata, Argentina
}

Entrar al mundo que nos propone Baricco ${ }^{1}$ en The Game es sumergirse en un verdadero juego, vertebrado por la lógica del ultramundo digital que, a través de guiños de complicidad y reconocimiento, interpela a un lector que acepta convertirse en un gamer. En el recorrido, nos obliga a pensar en las múltiples revoluciones que involucran la paulatina, pero incesante, digitalización del mundo, las migraciones y "la mutación de consistencia de la experiencia” (p. 44) y, con ello, las revisiones profundas que el campo de la educación podría producir para generar experiencias de aprendizaje que den cuenta de los desafíos que plantean las novedosas formas de apropiación, producción y consumo de conocimientos en escenarios altamente diversificados.

El autor sorprende desde el inicio con un título original en inglés. Nos integra a un universo de significaciones en perspectiva contemporánea plurilingüe, que pone en jaque modelos tradicionales de concebir el conocimiento, la transmisión, el saber. La dimensión lúdica del texto crea la ilusión de estar dentro de un escenario del que no podemos escapar. Atrapados en The Game, el juego de las deconstrucciones comienza.

El punto de partida, la primera posición, nos encuentra frente a una pantalla de papel donde un username y una password adelantan la estructura de un texto que promete zambullirnos en una realidad virtual, diseñada en un entorno analógico. Este doble entorno nos remite, inmediatamente, a la estructura escolarizada que oscila entre dos mundos aparentemente antagónicos pero que aún tienen la capacidad de vivir juntos.

El tablero se despliega con una evocación a Los bárbaros, escrito hace apenas diez años. Recuerda un escenario en el que se creía que la llegada de las computadoras personales, los teléfonos celulares y

1 Licenciado en Filosofía, Alessandro Baricco (1958) es novelista, ensayista, dramaturgo y periodista italiano. Autor, entre otras obras, de la novela Seda (1996) y Los bárbaros. Ensayo sobre la mutación (2008) que inspira el comienzo de la discusión de este libro. los dispositivos digitales anunciaban un apocalipsis, el fin de la belleza del mundo, el fin de los maestros y el fin de las instituciones educativas. Sin embargo, la revolución fue irreversible y en poco tiempo los nuevos programas no solo cambiaron nuestra percepción del entorno sino la concepción de la realidad. Bajo la idea de humanidad aumentada, y como herederos de los videojuegos, somos transportados a zonas desconocidas que parecen abrirse camino hacia una vida más sencilla, lúdica y agradable, escondiendo bajo sus pliegues la complejidad tecnológica.

En esa complejidad, encogida en la apariencia de simplicidad, las instituciones educativas insisten en sobrevolar la superficie conservando las rutas únicas, las estructuras formales, los lugares comunes fuertemente burocratizados, rutinarios, volviendo al plano de dos dimensiones aquello que -nos deja entrever Baricco- es tridimensional.

En un trabajo arqueológico, el autor develará los restos fósiles de una revolución "cuyo origen y propósito no conocemos con exactitud" (p. 19). Nos conducirá a través del juego por mapas de la insurrección digital y de los gestos simples que permiten visibilizar los abruptos cambios que, en menos de veinte años, anidaron en nuestras prácticas cotidianas. Así se presenta, entonces, así nos habilita su username y nos susurra su clave de acceso.

En la cartografía, Baricco adelanta que, contra todo sentido común, los cambios que percibimos como una revolución tecnológica son, en realidad, producto de una revolución mental, de un hartazgo de los jóvenes del siglo XX sobre el modo de ver y hacer en el mundo, "una reivindicación colectiva y no una degeneración imprevista del sistema” (p. 19). No se trata de una cuestión puramente técnica, sino de las ruinas de los arquetipos y de las verdades de una sociedad que debía ser pensada de otra manera y donde -en movimientos contraculturales- los jóvenes sintieron la urgencia de inventar el futuro. 
En este encuentro de movimientos rupturistas entre el sujeto y la cultura, Baricco nos permite volver hacia la mirada educativa y pensar en alternativas didácticas no instrumentales que recuperen los saberes de los jóvenes, el nomadismo de las ideas y que den cuenta de la necesidad de ser permeables a espacios contrahegemónicos. Baricco juega y, aunque sin proponérselo, nos fuerza a pensar la educación, la enseñanza y la innovación en un plano que rompe con las jerarquías del conocimiento, con la rigidez del tratamiento de los contenidos y anuncia la existencia de "un texto libre de las cadenas de la linealidad. Un texto compuesto en telaraña" (p. 84), hipermedial e hipertextual, donde en lugar de enviar $a$, debamos navegar con.

Tenemos usuario, ahora debemos introducir la password. El autor rompe el tablero y afirma que "las revoluciones tecnológicas, por muy fantásticas que puedan ser, no suelen producir, de forma directa, una revolución mental" (p. 31). La clave de acceso es, entonces, "revoluciones mentales" y tendremos que leer todo el ensayo en esa sintonía, admitiendo que, a pesar de las muchas revoluciones que cambian el mundo, son pocas -dirá Baricco- las que cambian a las personas radicalmente y su modo de mirar, interactuar y conocer.

Comenzamos a percibir, junto al autor, que la revolución digital es parte, también, de una revolución mental en la que anida y que no ha cambiado algo sino todo. El texto nos obliga a mirar de cabeza y reparamos, bruscamente, en que "un nuevo tipo de inteligencia ha generado las computadoras" (p. 36) y no a la inversa. De este modo, Baricco nos permite advertir que los cambios en la educación son, también, antes mentales que tecnológicos. La revolución mental precede a todo.

Play, estamos en el juego. El autor propondrá un recorrido histórico en el que intentará develar las fuerzas que empujaron hacia la revolución digital. El juego estará armado a modo de columna vertebral, y dividido en una vértebra cero y tres grandes momentos: La época clásica, La colonización y The Game.

La vértebra cero, que Baricco sitúa en el Space Invaders, recupera la idea de un cuerpo que desaparece detrás de una pantalla como campo de juego. La primera migración es una mutación de la consistencia de la experiencia y, por primera vez en la historia, el paso del juego analógico al digital. Así detecta, junto a este desplazamiento, un cambio de postura física y mental. En el origen de la revolución digital, Baricco encuentra un tipo de postura, de movimiento y de actitud que podrá hallarse en los restos de lo que viene.
La época clásica que irá desde el Commodor 64 hasta Google nos permite comprender los movimientos mentales que van dando origen a los acontecimientos de la revolución digital. En esta parte del juego, el autor subraya un primer hito: "Internet y la Web son dos cosas diferentes". Construye la parte inicial de su cadena montañosa y afirma que primero fue la digitalización, luego las computadoras personales y, finalmente, el golpe maestro: poner en red todas las computadoras. De esta manera, comienza a sedimentarse un cierto modo de estar en el mundo saltándose las mediaciones de viejas élites. En esta época clásica, ya no se envía, se navega. Se instala una obsesión por el movimiento, con desmaterializaciones que permiten hacer todo más liviano y nómade. El tablero ha sido invertido.

Tras esta época comienza la colonización. Baricco da cuenta de la conquista de la Web, entrecruzando acontecimientos históricos con el nacimiento de Wikipedia, Linkedin y el primer smartphone que llega a las manos de las personas convirtiéndose en prótesis. Todo lo que viene es vertiginoso: Skype, Facebook, Instagram, Snapchat, YouTube, Twitter, el Kindle y algo que será -según el autor- "un caso interesante de matrimonio entre un medio de comunicación antiguo, la televisión, y un medio nuevo, los ordenadores" (p. 130): las series. Así, entre gestos y movimientos, colonizamos masivamente ese más allá inmaterial que Baricco denomina ultramundo, gracias al smartphone que incorpora la dimensión lúdica con formato de videojuego, al modo de los mitos fundacionales de la insurrección digital.

Estamos sumergidos en la posexperiencia que, dirá el autor, es un flujo contrapuesto a la rigidez y que resulta, por momentos, difícil y desestabilizadora. La colonización del ultramundo tiene algunas condiciones: el movimiento permanente y la desmaterialización de la experiencia, la ruptura con las mediaciones y la confianza en la postura hombre-teclado-pantalla que permite crear sentido, intensidad y vibración en red. Esta afirmación plantea, desde el punto de vista educativo, tensiones que interrogan sobre la significatividad de lo que sucede en las instituciones educativas y, aún más, sobre sus estructuras fuertemente inerciales y rutinarias, sus arquitecturas inmóviles y sus recorridos fijos y predecibles.

Llegamos casi al final de la columna vertebral que Baricco construye para el lector. Este periodo estará signado por las App y los smartphones que aligeran la postura hombre-teclado-pantalla. Los viejos softwares se transforman en aplicaciones accesibles, prácticamente gratuitas que permiten disolver rápidamente 
los límites físicos y psicológicos entre mundo y ultramundo.

A pesar de todos estos cambios, Baricco afirma que la insurrección digital no ha logrado sacar a la escuela de su letargo. Ese viejo dispositivo permanece apacible en su inmovilidad. Seguramente, "el día en el que a alguien se le ocurra renovar un poco los locales, lo primero que irá, directamente, a la basura serán la clase, la asignatura, el profesor de la asignatura, el curso escolar, el examen" (p. 231).

The Game es un modo de estar en el mundo, es anidar en la red de una humanidad aumentada que, en tanto abre posibilidades, redistribuye el poder y rompe viejos privilegios. Pero crea otros nuevos y comienza, también, a "fijar modelos, a amontonar riquezas, a imponer gustos y establecer reglas" (p. 252) y se convierte en un entorno donde va fraguándose ese agregado de "jugadores singulares" que Baricco llamará "individualismo de masas" (p. 219). El Game muestra grietas y algunos derrumbes subterráneos. Nos queda, ahora, subir de nivel en el juego.

En su Level Up, Alessandro Baricco recuperará lo que queda de la verdad, lo que queda del arte y, por supuesto, lo que queda por hacer. En el primero de los tópicos, reconoce que el Game es dinámico y abierto y, por este motivo, deslizar la verdad o producirla está al alcance de cualquiera. Por esta característica -afirmael Game ha modificado el diseño de la verdad y sugiere que de lo que se trata es de una "verdad-rápida" que sube a la superficie y se rediseña de forma aerodinámica, perdiendo exactitud, pero ganando síntesis y velocidad. Del arte nos quedan unas hermosas zonas fronterizas, bilingües: E-books, Netflix, Spotify nos dan acceso a esos mundos caros y reservados para pocos, difíciles de alcanzar y casi nunca interactivos. Estas zonas fronterizas permiten el acceso a lugares como teatros, museos o librerías que no hubiésemos podido visitar y, por supuesto, dan visibilidad a nuevos artistas y autores. Sobre el final, en lo que queda por hacer, bajo el título Contemporary Humanities, desarroIla veinticinco tesis en las que invita a poner en juego nuestras ideas sobre la humanidad y el humanismo dentro del Game.

Ahora llega el momento de volver a estudiar cada mapa, superponerlos, hakeando la linealidad y jugando con las posibilidades y, al final, "disfrutar sus resultados en [...] una acrobacia típica de la posexperiencia que podrán llevarla a cabo, tal vez, personas que hoy van a la enseñanza media y se pasan las horas jugando al Far Cry" (p. 255).

The Game es una oportunidad para poner en suspenso los modos tradicionales de concebir el surgimiento y el impacto de las tecnologías en la vida cotidiana y en las instituciones educativas. Sus ideas cuestionan la forma habitual de comprender la relación entre nuestras formas mentales y las innovaciones tecnológicas. Por ello, el campo de la educación se enriquece después de la lectura de estas páginas. Su autor nos deja entrever una condición de posibilidad y un imperativo que obliga a buscar la vértebra cero, a rediseñar las clases desde los ultramundos, desde las experiencias cambiantes y móviles.

La lectura de Baricco es una inspiración para buscar ambientes de aprendizaje que nos permitan ser migrantes, creadores, movernos con soltura entre dispositivos cada vez más sofisticados e incorporados a la anatomía humana. The Game aporta miradas alternativas que llevan a preguntarnos si es posible modificar el tablero, romper mediaciones, extender las experiencias, revisar profundamente nuestras prácticas de enseñanza para que transmuten en otras. Las tecnologías y la conectividad provocan, sin dudas, reconfiguraciones en las prácticas, pero este libro invita a ir un poco más allá y a preguntarnos de qué modo producir, de manera colectiva, movimientos y vibraciones, sismos subterráneos que reverberen en la superficie.

\section{Referencias}

Baricco, A. (2008). Los bárbaros. Ensayo sobre la mutación. Buenos Aires: Anagrama 